\title{
Study on the characteristics of horseshoe vortex system upstream of an upright square cylinder subjected to a dam-break flow
}

\author{
C. Lin ${ }^{1}$, Y.-C. Su ${ }^{1}$, P.-H. Yeh ${ }^{1}$ \& R. V. Raikar ${ }^{2}$ \\ ${ }^{1}$ Department of Civil Engineering, \\ National Chung Hsing University, Taiwan \\ ${ }^{2}$ Department of Civil Engineering, KLES College of Engineering \\ and Technology, India
}

\begin{abstract}
The velocity-field characteristics of time-dependent horseshoe vortex flow at the juncture of an upright square cylinder and a base plate, which is subjected to an unsteady dam-break flow, were investigated experimentally. Flow visualization techniques and high-speed particle image velocimetry (HSPIV) were used to explore both qualitatively and quantitatively the unsteady feature of the flow field of horseshoe vortex structure at the juncture of square cylinder and base plate, respectively.

The characteristics of the evolutionary horseshoe vortex system were analyzed, including the position of primary vortex core, down-flow velocity profile and the circulation of primary horseshoe vortex. The results show that air bubble entrains into the vortex centre in front of the model only in the cases with model-width varying from $3.0 \mathrm{~cm}$ to $5.0 \mathrm{~cm}$. When the model width increases, the bubble entraining to the vortex centre occurs earlier. The results also show that the down-flow velocity profiles are highly affected by the vortex structures. The relationship between flow characteristics and the time duration after the gate is open is obtained by using regression analysis. The similarity of the position of the down-flow is presented with dimensionless length and dimensionless velocity as length and velocity scales, respectively.

Keywords: dam-break flow, horseshoe vortex, flow visualization technique, highspeed particle image velocimetry, air entrainment.
\end{abstract}




\section{Introduction}

Flood induced by the sudden collapse of a loose-soil dam (e.g., dam formed by massive land-slide of hill slope) can be termed dam-break flow and characterized by the generation of shock water waves passing very torrentially through river valley. The high speed flood then inundates most low-land area of valley and impinges upon most houses and infrastructures like bridge piers. These structures tend to be seriously damaged due to huge impacting force on and severe scour depth around them.

The flow field around an upright simplified obstacle is similar to that of a protruding cylinder mounted vertically on a horizontal base plate, being essentially a three-dimensional separated flow. The boundary layer upstream of the cylinder overcomes a strong adverse pressure gradient induced by the cylinder in the approaching flow direction. This results to a flow separation near the junction of the cylinder and the base plate. In the separated region, the developed vortex system which stretches around the cylinder is referred as a horseshoe vortex system due to its shape.

Schwind [1] pioneered in the study on a horseshoe vortex system upstream of a wedge placed in a wind tunnel. The periodical characteristics of the horseshoe vortex system was also noticed by Roper [2] and Dargahi [3], who used the hydrogen bubble method to study the flow structure around a circular cylinder. Baker [4] experimentally observed that as the Reynolds number $\operatorname{Re}_{D}\left(=U_{0} D / v=\right.$ $7.3 \times 10^{2} \sim 5.0 \times 10^{4}$, where $U_{0}$ denotes the free stream velocity, $D$ is the diameter of the circular cylinder, and $v$ represents the kinematic viscosity of fluid) increases under a laminar boundary layer flow, the number of horseshoe vortices grows and the vortex system becomes more complicated and unsteady. Thomas [5] conducted experiments on the water and wind flows around a circular cylinder in laminar and turbulent boundary layers in order to study the oscillating frequency of the periodic horseshoe vortex system. Baker [6] reported further results which related the oscillating frequency of the periodic vortex system with the variations of the boundary layer thickness.

Greco [7] recognized five flow regimes of the vortex system: steady horseshoe vortex system, oscillating vortex system, amalgamating vortex system, breakaway vortex system and transitional vortex system, for Reynolds numbers $\mathrm{Re}_{D}$ ranging from $3.5 \times 10^{2}$ to $3.2 \times 10^{4}$, using the hydrogen bubble method and a hot-film anemometry. Seal et al. [8] examined the instantaneous flow field around a square cylinder by using a high-density particle image velocimetry (PIV) and described the dominant mechanism of the oscillating cycle for the vorticity balance in a periodic breakaway vortex system for a Reynolds number $\left(\operatorname{Re}_{w}=U_{0} W / v\right.$, where $W$ denotes the width of upright square cylinder) of $7.6 \times$ $10^{3}$ and an aspect ratio of 1.3. Furthermore, Seal et al. [9] reported the interacting mechanism between the primary and corner vortices by presenting the detailed distributions and evolutions of the vortex vorticity for the oscillating cycle of a periodic breakaway vortex system. Lin et al. [10] used the PIV and flow visualization techniques to quantify the characteristics of a steady horseshoe vortex system at the junction of a vertical plate and a base plate for Reynolds 
numbers $\left(\operatorname{Re}_{w}\right)$ ranging from $2.0 \times 10^{2}$ to $1.2 \times 10^{3}$ and the aspect ratios $0.5 \sim 2$. Lin et al. [11] further carried out a comprehensive research on the horseshoe vortex system near the juncture of a vertical plate (with zero after-body dimensions) and a base plate in the laminar boundary layer flow. They used the flow visualization techniques, LDV, and PIV to determine the effect of the aspect ratio on the oscillating frequency of the horseshoe vortex system. The flow patterns were classified as five categories which are different from those in Schwind [1] and Greco [7] with some extension. Lin et al. [12] classified the horseshoe vortex system in the base of a vertical square cylinder as steady, periodical oscillatory and turbulent-like chaotic vortex systems using the flow visualization technique and time-domain spectral analysis for periodic breakaway vortex system having a Reynolds number $\left(\mathrm{Re}_{w}\right)$ of $2.25 \times 10^{3}$ and an aspect ratio of 2.0. Recently, Ballio et al. [13] and Simpson [14] put forward the comprehensive review on the horseshoe vortex systems.

The objective of the study is primarily to explore the unsteady flow field of the horseshoe vortex system in front of the square cylinder and base plate juncture, subjected to an unsteady dam-break flow. The flow visualization technique and PIV were used to study the characteristics of the unsteady horseshoe vortex system. The characteristics of the starting horseshoe vortex system were analyzed, including the position of primary vortex core, down-flow velocity profile and the circulation of primary vortex.

\section{Experimental setup}

Experiments were carried out in a re-circulating water channel having the dimensions of $14.0 \mathrm{~m}$ long, $0.50 \mathrm{~m}$ high and $0.25 \mathrm{~m}$ wide. The test section was fitted with glass-sided walls and glass bottom to facilitate optical access and was located at $8.5 \mathrm{~m}$ from the inlet of the water channel, where the gate was installed and could be lifted suddenly by the actuation control of a servo-motor. Sudden lifting of the gate can be used to generate the unsteady dam-break-like flow. The structures of horseshoe vortex were observed for an upright square-cylinder model having width ranging from $1.0 \mathrm{~cm}$ to $5.0 \mathrm{~cm}$ (see Table 1). The model was placed at a position of $66 \mathrm{~cm}$ downstream of the gate. The schematic diagram of the experimental set-up and coordinate system are shown in Fig. 1.

Flow visualization was performed to observe the temporal evolution of the vortical structure in the juncture area of both frontal face of square cylinder and base plate, using the particle trajectory photography. Aluminum powder particles having mean diameter $20 \mu \mathrm{m}$ were added into water as the tracers. A laser beam emitted from a $7 \mathrm{~W}$ argon-ion laser tube (Coherent Innova-90) was spread into a fan-shaped light sheet (about $1.0 \mathrm{~mm}$ thick) using a glass cylinder. The light

Table 1: $\quad$ Test conditions and the widths of the square cylinder.

\begin{tabular}{|c|c|c|c|c|c|}
\hline & Case A & Case B & Case C & Case D & Case E \\
\hline $\begin{array}{c}\text { Width, } W \\
(\mathrm{~cm})\end{array}$ & 1 & 2 & 3 & 4 & 5 \\
\hline
\end{tabular}




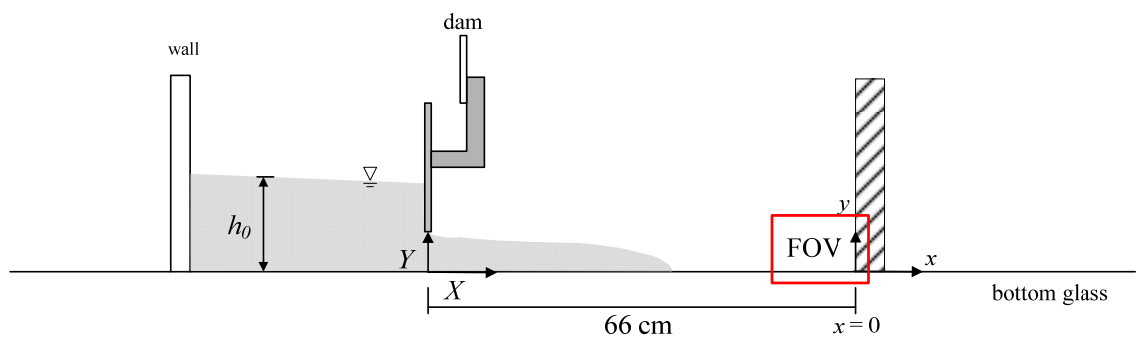

Side view

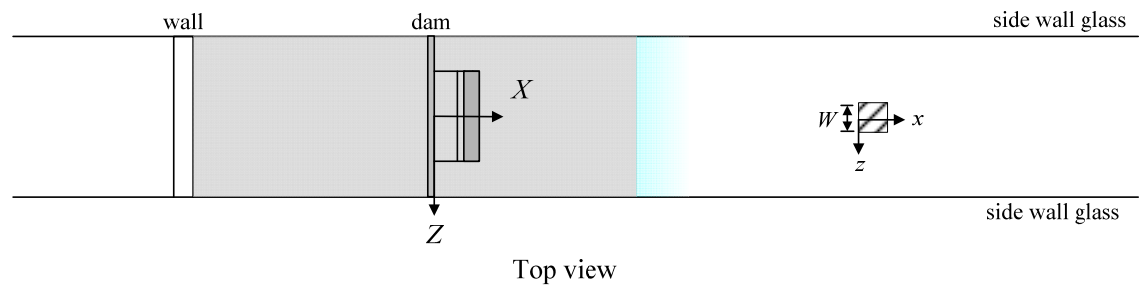

Figure 1: $\quad$ Experimental setup and coordinate system.

sheet was spread upwards through the glass bottom of the tank to illuminate the two-dimensional motion of the tracing particles in the plane of symmetry. A high-speed image camera (1024 × 1024 pixel, Vision Research V5.1) was used to film the brightened tracers.

A high-speed PIV system was used to obtain the entire velocity field of vortical structure taking place right ahead of the square cylinder. The light sheet optics consisted of a cylindrical lens and a spherical lens. With a certain combination of these lenses, the laser beam could be expanded into a fan-shaped light (about $1.0 \mathrm{~mm}$ thick) which was projected upwards into the plane of symmetry through the glass bottom of the tank. The aluminum powder particles (having mean diameter of $20 \mu \mathrm{m}$ ) being added into the water as tracers were illuminated by the light sheet. The image recording system was a 10 bit Complementary Metal-Oxide (CMO) high speed camera (Phantom V5.1) having resolution of $1024 \times 1024$ pixel and $1200 \mathrm{~Hz}$ maximum framing rate. Commercial software (TSI Insight) was used to process the PIV images and compute velocity vectors through two-frame cross-correlation. A multi-grid interrogation process starting at $32 \times 32$ pixels and ends at $16 \times 16$ pixels with $50 \%$ overlap were used in the processing.

\section{Results}

Figures 2-3 show the flow visualization images and corresponding PIV measurements in the juncture area of the frontal face of the square cylinder and base plate in different non-dimensional time $T$. The upstream water depth behind 
the gate $h_{0}$ in these figures is $15 \mathrm{~cm}$. It is seen that the approaching dam-break flow arrives the square cylinder at $T=4.9$ (Fig. 2(a)), and the flow field bifurcates along the frontal face of the square cylinder. A horseshoe vortex system gradually forms around the square cylinder (Fig. 2(b)). The horseshoe vortex rotates clockwise and creates down-flow velocity towards the toe of the cylinder (Fig. 2(c)). The position of the stagnation point continues to rise from $11 \mathrm{~mm}$ to $27 \mathrm{~mm}$ above the base plate.
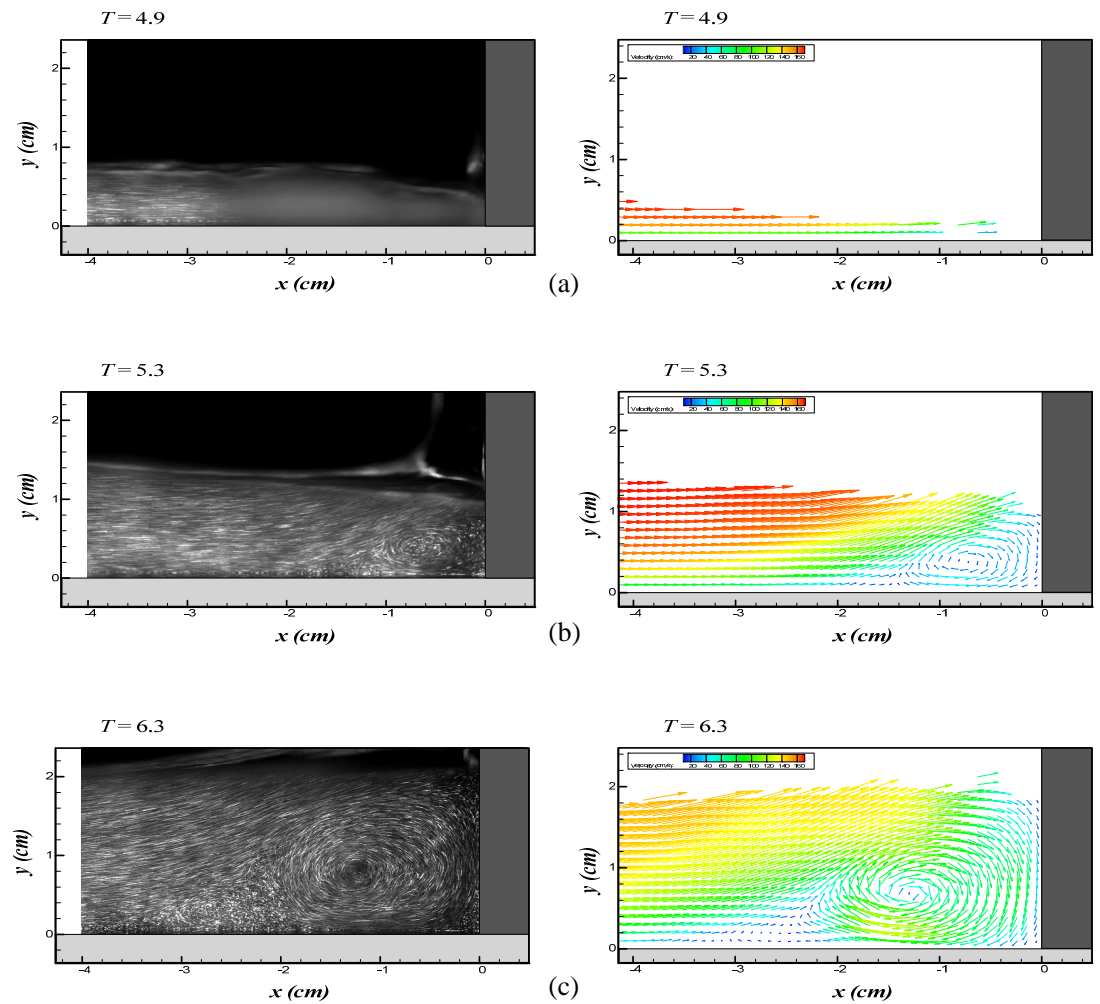

Figure 2: Flow visualization images and corresponding PIV measurements from $T=4.9-6.3$.

Figure 3 shows the evolution of the horseshoe vortex and its surrounding flow field from $T=6.8-8.3$. It shows that as the non-dimensional time $T$ increases, the size of the horseshoe vortex increases accordingly. Its centre (vortex core) also gradually rises and moves away from the cylinder. Finally, the horseshoe vortex system reaches a relatively stable state at about $T=6.8$. The figure also shows that air entrainment occurs at about $T=6.8$ (Fig. 3(a)). The entrainment starts from the two sides of the cylinder and gradually approaches the central axis of the frontal face. 

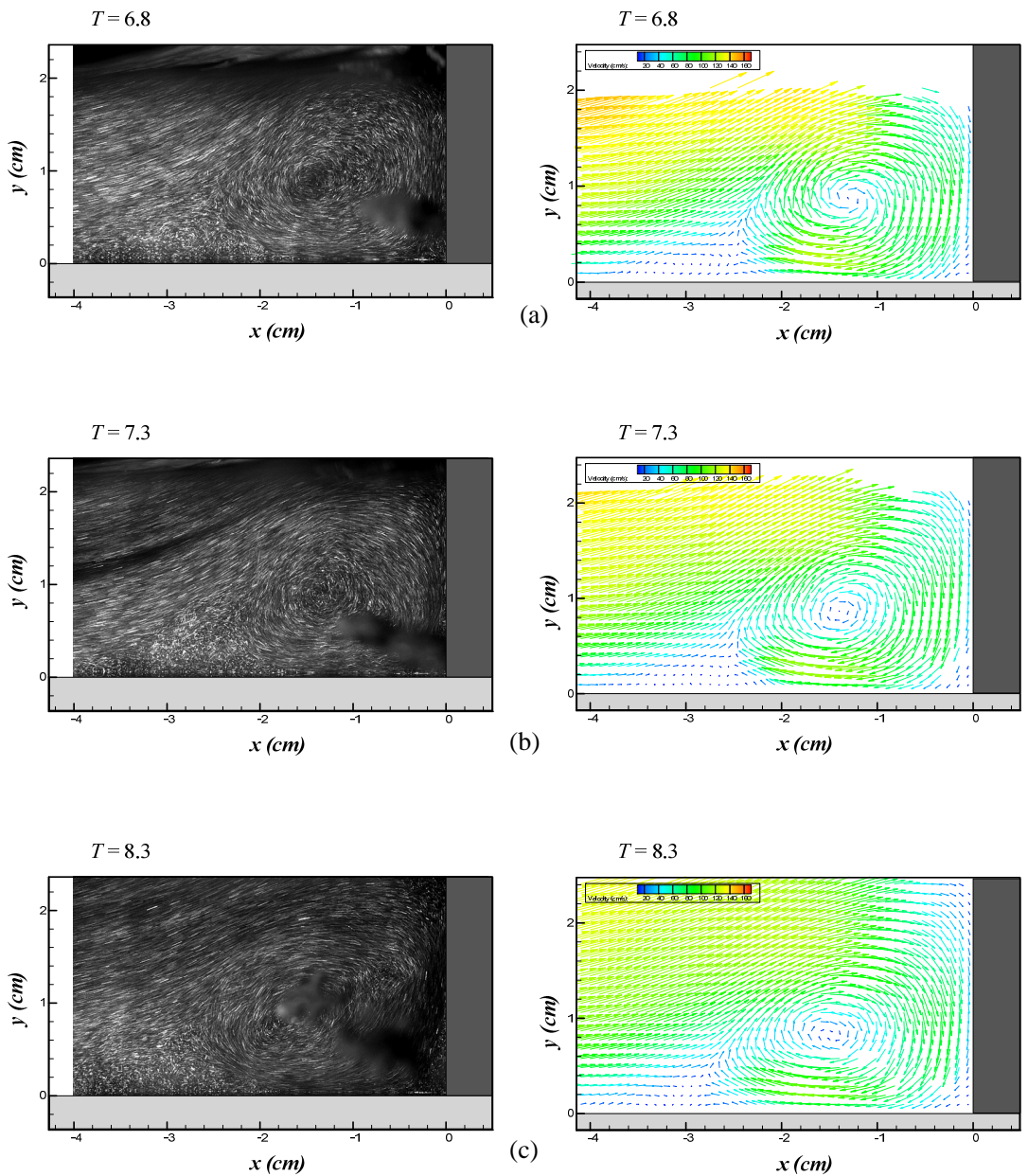

Figure 3: Flow visualization images and corresponding PIV measurements from $T=6.8-8.3$.

The instantaneous down-flow velocity profiles can be obtained from the phase-averaged velocity fields measured by PIV. One of the examples is shown in Fig. 4 for Case E with $W=5.0 \mathrm{~cm}$ and $T=5.5 \sim 8.5$. It can be seen clearly that, for each velocity profile the negative maximum down-flow velocity, $V_{d M}$ takes place at a specific position, $x_{v}$. If $x_{v}$ and $V_{d M}$ are considered as the representative length and velocity scales, respectively, then the velocity profiles shown in Fig. 4 can be non-dimensionalized. The corresponding result is shown in Fig. 5. It is surprisingly found that all of the non-dimensional velocity profiles collapse onto a single trend, demonstrating a promising similarity profile having the following equation form: 


$$
\frac{V_{d}}{V_{d M}}=-0.263-6.825\left(\frac{x}{x_{v}}\right)+20.331\left(\frac{x}{x_{v}}\right)^{2}-22.940\left(\frac{x}{x_{v}}\right)^{3}+9.971\left(\frac{x}{x_{v}}\right)^{4}
$$

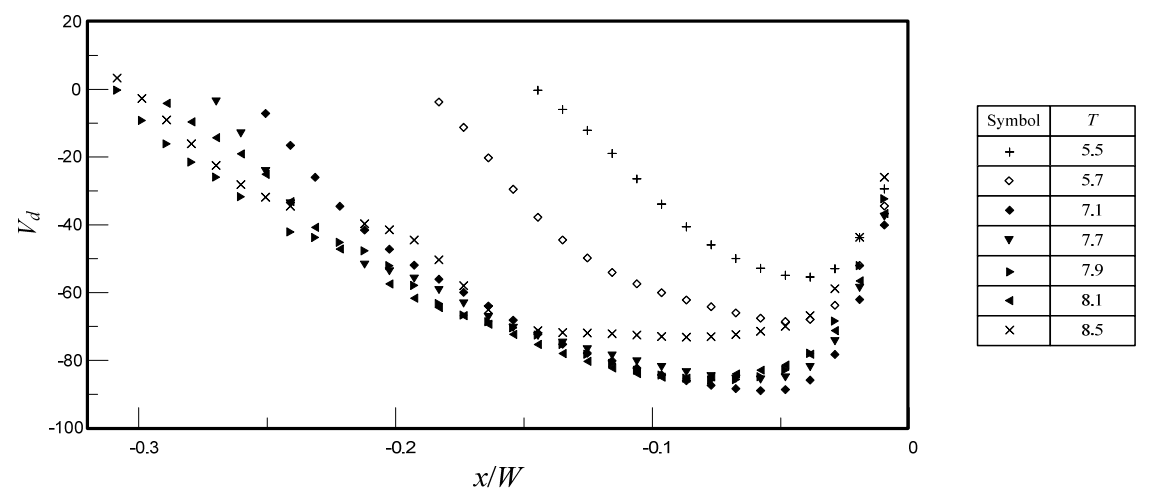

Figure 4: Temporal variation of down-flow velocity $V_{d}$ for Case $\mathrm{E}$ at different $x / W$ locations.

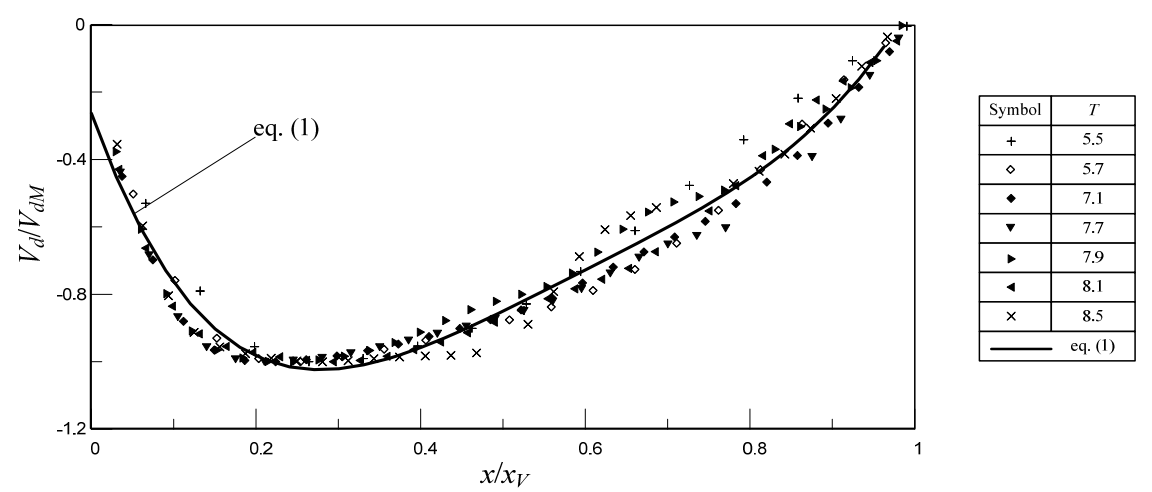

Figure 5: $\quad$ Similarity profile of down-flow velocity $V_{d}$ for Case E.

Similarly, the instantaneous peak vorticity maps of horseshoe vortex can be also obtained from the phase-averaged velocity fields using Stokes's theorem. One of the examples is shown in Fig. 6 for Case $E$ at non-dimensional time $T=6.3$. The temporal variations of the peak vorticity of horseshoe vortex are depicted in Fig. 7 for Cases $\mathrm{C}$ E with $T$ being changed from 5.2 to 6.8. Note that right at $T_{0}$ the dam-break flow impinges upon the upright square cylinder, and exactly at $T_{s}$ the horseshoe vortex reaches a relatively stable state. By choosing the non-dimensional time difference, $\left(T_{s}-T_{0}\right)$, and the ratio of peak vorticity $\Gamma / \Gamma_{s}$, in which $\Gamma_{s}$ is the peak vorticity when $T=T_{s}$, as the representative time and vorticity scales, the non-dimensional form of Fig. 7 can be illustrated in Fig. 8 for Cases $\mathrm{C} \sim \mathrm{E}$. It is worthy of mentioning that, although the data points seem to 


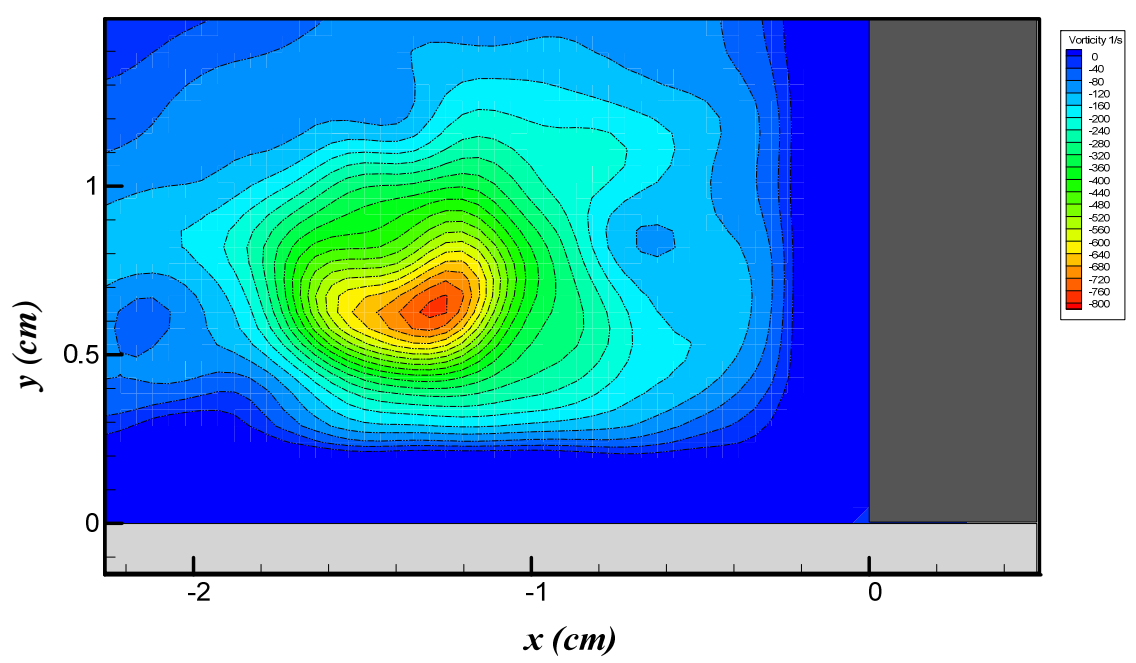

Figure 6: Instantaneous peak vorticity of Case $\mathrm{E}$ at $T=6.3$.

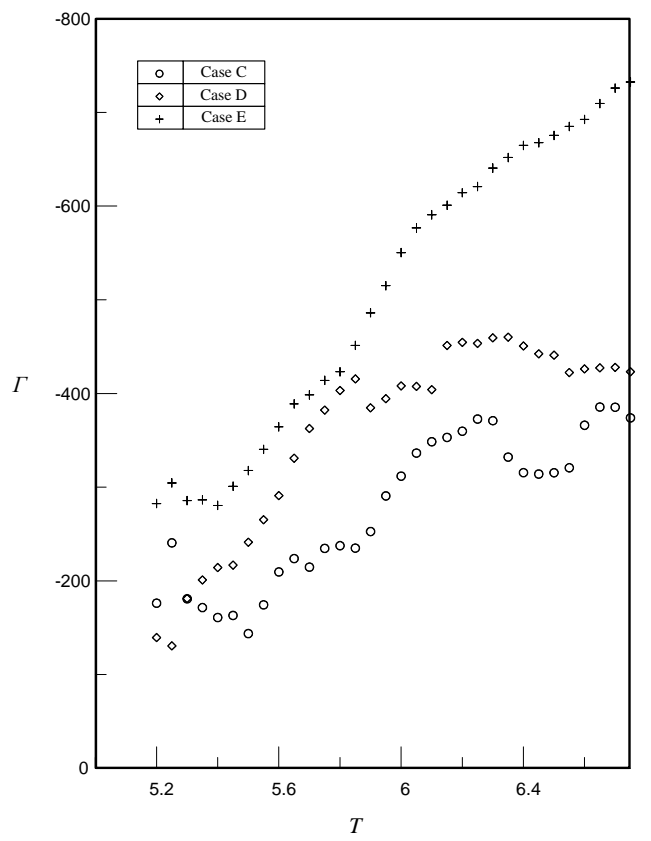

Figure 7: $\quad$ Temporal variations of peak vorticity for different cases. 


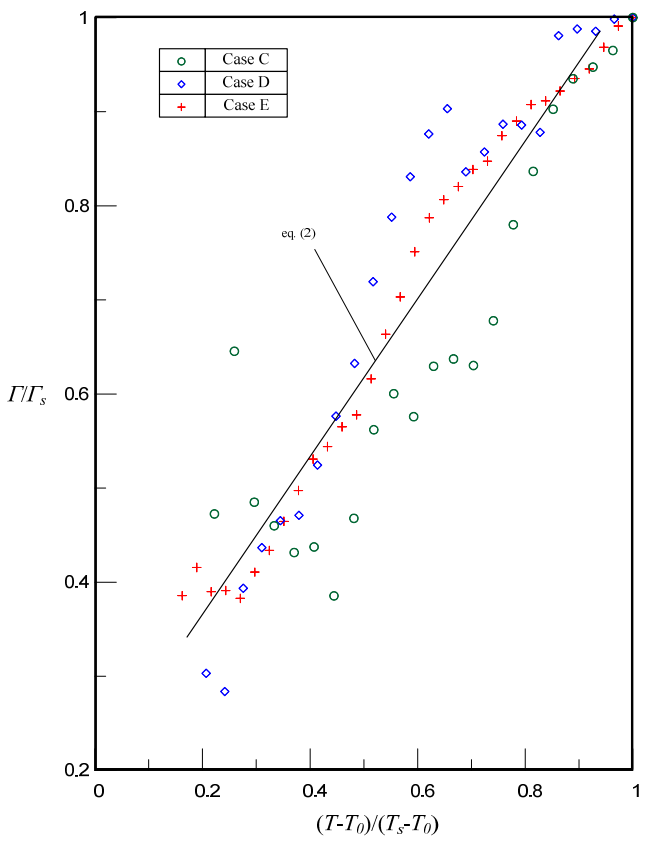

Figure 8: $\quad$ Non-dimensional peak vorticity of different cases.

be somewhat scattering, the relationship between the non-dimensional vorticity $\Gamma / \Gamma_{\mathrm{s}}$ and the non-dimensional shifted-time parameter $\left(T-T_{0}\right) /\left(T_{s}-T_{0}\right)$ demonstrates a seemingly linear trend:

$$
\frac{\Gamma}{\Gamma_{s}}=0.844+0.202\left(\frac{T-T_{0}}{T_{s}-T_{0}}\right)
$$

\section{Conclusions}

A time-dependent horseshoe vortex system at the juncture of an upright square cylinder and a base plate was investigated experimentally. The vortex system was subjected to an unsteady dam-break flow in the experiments. Flow visualization techniques and high-speed particle image velocimetry (HSPIV) were used to explore both qualitatively and quantitatively the unsteady feature of the flow field of horseshoe vortex structure at the juncture of square cylinder and base plate. The results show that air bubble entrains into the vortex centre in front of the model only in the cases with model-width varying from $3.0 \mathrm{~cm}$ to $5.0 \mathrm{~cm}$. When the model width increases, the bubble entraining to the vortex centre occurs earlier. The results also show that the down-flow velocity profiles are highly affected by the vortex structures. The relationship between flow 
characteristics and the time duration after the gate is open is obtained by using regression analysis. The similarity of the position of the down-flow is presented with dimensionless length and dimensionless velocity as length and velocity scales, respectively.

\section{References}

[1] Schwind, R. (1962). "The three-dimensional boundary layer near a strut." Gas Turbine Laboratory Rep., Massachusetts Institute of Technology, Cambridge, MA.

[2] Roper, A. T. (1967). A cylinder in a shear flow. Ph.D. Thesis, Colorado State University, Fort Collins, CO.

[3] Dargahi, B. (1989). "The turbulent flow field around a circular cylinder." Exp. in Fluids, 8, 1-12.

[4] Baker, C. J. (1979). "The laminar horseshoe vortex.” J. Fluid Mech., 95, 347-367.

[5] Thomas, S. W. (1987). “The unsteady characteristics of laminar juncture flow.” Phys. Fluids, 30(3), 283-285.

[6] Baker, C. J. (1991). "The oscillation of horseshoe vortex system.” J. Fluid Eng., 113(3), 489-495.

[7] Greco, J. J. (1990). The flow structure in the vicinity of a cylinder-flat plate junction: flow regime, periodicity, and vortex interactions. M.S. Thesis, Dept. of Mechanical Engineering and Mechanics, Lehigh Univ., Bethlehem, PA.

[8] Seal, C. V., Smith, C. R., Akin, O., and Rockwell, D. (1995). "Quantitative characteristics of a laminar unsteady necklace vortex system at a rectangular block-flat plate juncture.” J. Fluid Mech., 286, 117-135.

[9] Seal, C. V., Smith, C. R., and Rockwell, D. (1997). "Dynamics of vorticity distribution in wall junctures.” AIAA J., 35(6), 1041-1047.

[10] Lin, C., Ho, T. C., Chang, K. T., and Shieh, S. J. (2001). "The characteristics of horseshoe vortex near the juncture of a vertical plate at low Reynolds number.” J. Eng. of National Chung Hsing Univ., 12(1), 115.

[11] Lin, C., Chiu, P. H., and Shieh, S. J. (2002). "Characteristics of horseshoe vortex system near a vertical plate-base plate juncture.” Exp. Therm. Fluid Sci., 27(1), 25-46.

[12] Lin, C., Lai, W. J., and Chang, K. A. (2003). "Simultaneous particle image velocimetry and laser Doppler velocimetry measurements of periodical oscillatory horseshoe vortex system near square cylinder-base plate juncture.” J. Eng. Mech., 10(1), 1173-1183.

[13] Ballio, F., Bettoni, C., and Franzetti, S. (1998). “A survey of time-averaged characteristics of laminar and turbulent horseshoe vortices.” J. Fluid Eng., 120(4), 233-242.

[14] Simpson, R. L. (2001). “Junction flows.” Annu. Rev. Fluid Mech., 33, 415443. 